\title{
Fatores que Facilitam e Dificultam a Atividade de Educação Permanente em Saúde
}

\author{
Rafael Silvério de Moraes ${ }^{1}$, Magali Aparecida Alves de Moraes², Elza \\ de Fátima Ribeiro $\mathrm{Higa}^{2}$, Carlos Alberto Lazarini ${ }^{2}$, Adriana Avanzi \\ Marques Pinto ${ }^{3}$
}

\author{
${ }^{1}$ Discente do Mestrado Profissional Ensino em Saúde pela Faculdade de Medicina de \\ Marília - Famema, Brasil | rafasilveriopdi@hotmail.com | http://orcid.org/0000-0002-4526- \\ 4275 \\ 2 Docente da Famema, Brasil | dmagalimoraes@hotmail.com; hirifael@gmail.com; \\ lazarini@famema.br | http://orcid.org/0000-0001-5888-1638; http://orcid.org/0000-0001- \\ 5772-9597; https://orcid.org/0000-0003-3010-4436 \\ ${ }^{3}$ Docente da Fundação Educacional do Município de Assis - Fema, Brasil \\ driavanzi1981@gmail.com | https://orcid.org/0000-0002-6055-6145
}

\begin{abstract}
Resumo: Introdução: A Educação Permanente em Saúde (EPS) é um processo educativo que articula o serviço, o ensino, a saúde e a docência, colaborando para o crescimento profissional, o controle social e a gestão. Objetivo: Identificar os fatores que facilitam e dificultam as atividades de EPS desenvolvidas pela equipe multiprofissional nas Unidades de Saúde da Família (USF). Metodologia: Pesquisa qualitativa fundamentada na Hermenêutica Dialética. Participaram 50 profissionais, das equipes multiprofissionais das USF, do interior paulista no Brasil, por meio de entrevistas semiestruturadas. Resultados: A análise dos sentidos evidenciou duas categorias analíticas: fatores que facilitam as atividades de EPS reuniões de equipe, trocas de informações e diferentes saberes, participação da população, colaboradores, coordenadores, da gestão e da equipe multiprofissional; fatores que dificultam as atividades de EPS - incompreensão do processo da EPS, falta de interesse de alguns colaboradores, desconsideração das necessidades dos profissionais pelos gestores e falta de participação da equipe multiprofissional na EPS. Conclusões: A abordagem qualitativa da pesquisa e a fundamentação teórica sustentada pela Hermenêutica-Dialética possibilitou à apresentação dos fatores que apoiam e impedem as atividades de EPS desenvolvidas pela equipe multiprofissional nas USF e que podem subsidiar as adequações necessárias.
\end{abstract}

Palavras-chave: Educação Permanente; Educação Permanente em Saúde; Equipe de Assistência ao Paciente; Estratégia Saúde da Família.

\section{Factors that Facilitate and Difficult the Permanent Health Education Activity}

Summary: Introduction: Permanent Education in Health (EPS) is an educational process that articulates service, teaching, health and teaching, collaborating for professional growth, social control and management. Objective: To identify the factors that facilitate and hinder the EPS activities developed by the multiprofessional team in the Family Health Units (FHU). Methodology: Qualitative research based on Dialectic Hermeneutics. Fifty professionals participated, from the multiprofessional teams of the $\mathrm{FHU}$, from the interior of São Paulo in Brazil, through semi-structured interviews. Results: The analysis of the senses showed two analytical categories: factors that facilitate the activities of EPS - team meetings, exchanges of information and different knowledge, participation of the population, collaborators, coordinators, management and the multidisciplinary team; factors that hinder EPS activities lack of understanding of the EPS process, lack of interest from some employees, disregard of the professionals' needs by managers and lack of participation by the multiprofessional team in EPS. Conclusions: The qualitative approach of the research and the theoretical foundation supported by Hermeneutics-Dialectics enabled the presentation of the factors that support and prevent the EPS activities developed by the multiprofessional team in the USF and that can subsidize the necessary adjustments.

Keywords: Permanent Education; Permanent Health Education; Patient Assistance Team; Family Health Strategy. 


\section{Introdução}

O termo Educação Permanente (EP) surgiu na França em 1955 pela primeira vez e foi oficializado no ano seguinte por meio de um documento desenvolvido pelo Ministério da Educação sobre a reforma e o prolongamento do ensino público. A Organização Pan Americana de Saúde (OPAS) na década de 1980 divulgou o Programa de Desenvolvimento de Recursos Humanos e a Educação Permanente em Saúde (EPS) (Lemos, 2016; Organizacion Panamericana de La Salud, 1989).

Para Ausubel, a aprendizagem significativa é um processo que ocorre por meio de uma informação nova com uma condição específica de conhecimento, definida como conceito subsunçor. Nesse sentido, a aprendizagem significativa ocorre quando a informação nova se ancora em concepções ou relevantes proporções, pré-estabelecidas na organização cognitiva do indivíduo (Ausubel, 1982).

A Estratégia Saúde da Família (ESF) criada em 1994 pelo Ministério da Saúde (MS), inicialmente como Programa Saúde da Família (PSF), abrange os princípios da Atenção Primária à Saúde (APS) desenvolvidos na Conferência de Alma Ata, interligada nas premissas aderidas pela formulação do Sistema Único de Saúde (SUS), como estratégia de reorientar os serviços de atenção à saúde (Soratto, Pires, Dornelles, \& Lorenzetti, 2015).

Nesse sentido, a ESF foi constituída como uma proposta de mudança do modelo tradicional de assistência em saúde, caracterizado como um modelo tecnicista, fragmentado, centrado na doença e hospitalocêntrico, visto que, o mesmo demonstrou ser incapaz de atender, com competência e justiça, as necessidades de saúde da população. No entanto, em respeito à realização do trabalho em saúde, ocorre uma mudança na centralidade da hegemonia de uma determinada categoria profissional para uma proposta de trabalho em equipe multiprofissional atuando com uma perspectiva interdisciplinar (Soratto, Pires, Dornelles, \& Lorenzetti, 2015).

Portanto, cada Unidade de Saúde da Família (USF) é constituída por uma equipe multiprofissional com estratégia prioritária de atenção à saúde que preconiza à reorganização da Atenção Básica (AB) no Brasil, em consonância aos preceitos do SUS (Portaria n. 2.436, 2017).

Nesse sentido, considerando que a PNEPS apoia a gestão do SUS e que a USF é um cenário propício para tal, questiona-se: Quais os fatores que facilitam e dificultam as atividades de EPS desenvolvidas pela equipe multiprofissional nas USF?

\section{Objetivo}

Identificar os fatores que facilitam e dificultam as atividades de EPS desenvolvidas pela equipe multiprofissional nas USF.

\section{Metodologia}

\subsection{Tipo de Pesquisa}

Pesquisa de campo com abordagem qualitativa fundamentada na Hermenêutica Dialética. A pesquisa qualitativa utiliza o estudo da história, das representações, das relações, das crenças, das opiniões e das percepções, o significado dos sentidos que os indivíduos produzem em relação a como vivem, sentem, pensam e elaboram seus produtos e a si mesmos. Preocupa-se com questões da realidade que não podem ser quantificadas e no entendimento das relações sociais, de modo holístico, dinâmico e individual da experiência humana (Minayo, 2014; Gerhardt, 2009). 
No estudo de campo o pesquisador coleta os dados diretamente do ambiente, levando em consideração as condições naturais em que ocorrem os fenômenos, sendo observados de forma direta, sem manuseios e intervenções por parte do pesquisador, abrangendo desde levantamentos mais descritivos, até estudos analíticos (Severino, 2016).

Hermenêutica é uma palavra derivada do grego "hermeneuein", que significa expressar, interpretar e traduzir. Os textos e as expressões são os materiais que constituem suas fontes com o objetivo de alcançar a compreensão dos seus significados. Foi desenvolvida na Antiguidade, pela distinção entre significados alegóricos e literais, tendo como exemplo, a interpretação da Septuaginta por Filon de Alexandria, na leitura de Homero e da mitologia grega (Gilhus, 2016).

A Hermenêutica procura demonstrar para o mundo por meio da sua historicidade e finitude, o seu caráter interpretativo. Tendo em vista a responsabilidade central pelo conhecer, pensar para a vida prática, não permitindo ser interrogada por outras experiências, como por exemplo, a artística (Hermann, 2002).

Dialética é uma palavra derivada do grego "dialektiké", que era utilizada em sua origem para designar a arte de discutir, do diálogo, e seu adjetivo "dialektikós", designa a tudo que se refere à discussão. A sua história foi caraterizada por duas fases. De Pré-socráticos até Hegel como "Antiga", de Hegel até os dias atuais como "Moderna" (Minayo, 2014; Bettoni, 2001).

A combinação entre Hermenêutica e Dialética foi descrita primeiramente por Habermas (1978), em compreender que, por meio do pensamento é realizado a síntese dos processos críticos e compreensivos (Minayo, 2014).

A Hermenêutica compreende o sentido que é produzido pela comunicação entre os seres humanos, tendo a linguagem como seu núcleo principal. Ela trabalha com a comunicação do senso comum e da vida cotidiana, abordando alguns preceitos. Sendo eles, a) o homem como um ser finito e histórico se complementa por meio da comunicação, b) necessidade de compreender a história e o contexto do homem, pois sua linguagem é limitada com ocupação no espaço e no tempo (Minayo, 2014).

Diferentemente da Hermenêutica, a Dialética busca por meio da linguagem, dos fatos, nos símbolos, e na cultura, os núcleos que estão incompreensíveis para produzir uma crítica esclarecida sobre eles. $\mathrm{Na}$ articulação entre a Hermenêutica e Dialética, Habermas diz que, o mesmo entendimento que esclarece, reúne e compreende, também protesta, critica e dissocia, portanto, a Hermenêutica busca compreender os sentidos, e a dialética produz uma atitude crítica (Minayo, 2014; Minayo, \& Deslandes, 2013).

\subsection{Participantes, Local de Pesquisa e Instrumento de Coleta de Dados}

A proposta do estudo foi de entrevistar 69 profissionais das equipes multiprofissionais das USF, sendo 36 graduados, caracterizando $100 \%$ dos profissionais de 12 USF e 33 profissionais não graduados por meio da amostragem intencional o que constituiu em 1/3 da população de (99), em um município situado no interior do Estado de São Paulo - Brasil, cidade com população estimada em 2020 de 105.087 habitantes (IBGE, 2020). De acordo com o histórico de cobertura da $A B$, esse município possui 13 equipes de Saúde da Família cadastradas no sistema do Departamento de Atenção Básica (DAB), sendo uma equipe em zona rural, totalizando $52,25 \%$ de cobertura populacional no período de janeiro a outubro de 2020 e cada equipe conta com um enfermeiro (E-Gestor, Informação e Gestão da Atenção Básica, 2020). Os profissionais da USF zona rural, não foram autorizados a participar da pesquisa pelo departamento responsável do município.

A pesquisa utilizou os seguintes critérios de inclusão: experiência mínima de 06 meses na ESF e os de exclusão: profissionais de licença maternidade, médica e licença prêmio. 
O instrumento de coleta de dados foi constituído por um roteiro de entrevista semiestruturada. Nessa modalidade o roteiro semiestruturado desenvolveu diversos indicadores considerados suficientes e fundamentais, por meio de tópicos que proporcionaram a abrangência das informações desejadas. Esses tópicos devem servir apenas como lembretes, tendo em vista que, o investigador deverá memorizá-los na medida do possível, servindo como guia e orientação para o desenvolvimento da interlocução. Os tópicos precisam ser desenvolvidos de forma flexível para as conversas, permitindo adquirir novas questões e temas dados pelo interlocutor (Minayo, 2014).

As entrevistas foram agendadas com contatos prévios, por meio de ligações telefônicas, em dias e horários estabelecidos pelos profissionais. O pesquisador foi até as USF convidando a equipe multiprofissional para que tomassem ciência da pesquisa e os que concordaram foram entrevistados conforme instrumento elaborado para coleta de dados.

Essa pesquisa foi aprovada pelo Comitê de Ética em Pesquisa da Faculdade de Medicina de Marília (FAMEMA) que envolve seres humanos, sob o parecer número 4.105 .188 e do Certificado de Apresentação de Apreciação Ética (CAAE) 26595119.7.0000.5413. Os participantes foram orientados sobre a pesquisa e seus objetivos e assinaram o Termo de Consentimento Livre e Esclarecido (TCLE).

\subsection{Análise de Dados}

Os dados obtidos foram analisados segundo o referencial teórico da HermenêuticaDialética. Sendo uma técnica proposta como um caminho do pensamento, que compreende o como fazer, e o como pensar a análise de dados (Cardoso, Santos, \& Alouffa, 2015).

A análise de sentidos segundo referencial teórico da Hermenêutica-Dialética é realizada por meio de três fases, que se organizam em: Ordenação dos dados, Classificação dos dados e Análise final (Minayo, 2014).

A Ordenação dos dados abrange as entrevistas como documentos institucionais e populares, e o conjunto de material de análise, relacionados ao tema. Essa etapa está composta por: (I) transcrever as gravações das entrevistas; (II) realizar a releitura do material; (III) organizar os relatos em ordem, no que pressupõe um começo de classificação; (III) organizar os dados de análise em ordem, conforme a proposta analítica. Esse trabalho é compreendido como um processo da Hermenêutica, no momento em que engloba o material empírico em relação as compreensões dos entrevistados (Minayo, 2014).

A segunda etapa é realizada pela leitura transversal dos conjuntos e subconjuntos em sua totalidade. Esse processo é desenvolvido por meio dos recortes de cada documento e entrevista, em unidade de sentido, por tópicos de informações, temas, ou estruturas relevantes. Em primeira instância o critério de classificação pode ser por variáveis teóricas ou empíricas, que já foram construídas pelo pesquisador. Esse processo classificatório faz com que o pesquisador separe unidades de sentido, categorias ou temas, colocando junto as partes idênticas, buscando compreender as conexões existentes entre elas, e sendo guardadas em gavetas ou códigos (Minayo, 2014).

Portanto, quando o pesquisador finalizar seu primeiro esforço em que muitas gavetas foram realizadas, ele partirá para uma segunda atividade, em realizar a redução de suas classificações, organizando em número menor de unidades de sentido, procurando interpretar e compreender aquilo que foi destacado como mais importante e significativo pelo grupo estudado. A gavetas são reagrupadas em categorias centrais, formando uma lógica unificadora (Minayo, 2014).

As etapas de ordenação e classificação dos dados irão pleitear uma mudança profunda sobre o material empírico, que deve ser considerado como ponto de partida e chegada da interpretação e compreensão. Após a análise final, o pesquisador apresentará a exposição da comunicação dos dados da pesquisa, o produto da investigação (Minayo, 2014). 
O relatório final da pesquisa deve ser representado como uma síntese, em que o objetivo impregna e reveste o texto de forma completa, levando em consideração que a forma de realizar a síntese deve contemplar a proposta do trabalho (Minayo, 2014).

Para garantir os aspectos éticos, na apresentação dos resultados, os participantes foram assim codificados: "ACS", em referência a "Agente Comunitários de Saúde", seguidos de sequência numérica crescente, por exemplo, "ACS1", até "ACS13"; "ASB", em referência a "Auxiliar em Saúde Bucal", seguidos de sequência numérica crescente, , "ASB1", até "ASB6"; "AE", em referência a "Auxiliar em Enfermagem", seguidos de sequência numérica crescente, "AE1" até "AE9"; "DENT", em referência a "Dentista", seguidos de sequência numérica crescente, "DENT1" até "DENT5"; "ENF", em referência a "Enfermeiro", seguidos de sequência numérica crescente, "ENF1" até "ENF11"; e "MED", em referência a "Médico", seguidos de sequência numérica crescente, "MED1" até "MED6".

\section{Resultados e Discussão}

De uma população de 69 profissionais, nove graduados e dez não graduados recusaram a participar da pesquisa. Desse modo, foram realizadas 50 entrevistas com os integrantes da equipe multiprofissional das USF.

Os dados sociodemográficos demonstraram predomínio do sexo feminino $(86 \%)$, da faixa etária entre 43 e 53 anos (42\%) e da atuação enquanto ACS (26\%). O tempo de atuação na ESF foi o mesmo para 1 a 10 anos e 11 a 21 anos, ambos com 38\%. Já a escolaridade mais prevalente foi o ensino superior (58\%).

A análise dos sentidos sobre os dados obtidos evidenciou duas categorias analíticas: fatores que facilitam as atividades de EPS e fatores que dificultam as atividades de EPS.

\subsection{Fatores que Facilitam as Atividades de Educação Permanente em Saúde}

A presente categoria analítica desvelou os fatores que facilitam as atividades de EPS: reuniões de equipe, trocas de informações e diferentes saberes, participação da população, colaboradores, coordenadores, da gestão e da equipe multiprofissional.

"Acredito que as reuniões de equipe é um facilitador, porque ali estamos todos reunidos e podemos trocar ideias, então, é um espaço muito importante" (ACS2).

"É essa troca de informações em áreas e saberes diferentes, eu acredito que isso facilita a Educação Permanente na Estratégia. $\mathrm{E}$ o que deixaria muito mais fácil para desenvolver essa Educação Permanente em Saúde é a colaboração tanto da população, quanto dos próprios colaboradores, coordenadores e da gestão" (AE1).

"É a própria equipe aceitar, porque se o profissional tem resistência a qualquer tipo de situação imposta, ele não vai participar e nem colaborar, e a gente não tem esse problema na nossa equipe, todo mundo gosta de participar e entende que isso é um crescimento e um conhecimento a mais, é um processo de trabalho e uma prática educativa que só serve para crescer e atender com qualidade a nossa população [...]" (ENF2).

"Acho que são as capacitações que acontecem durante as nossas reuniões de equipe, que são todas as quintas-feiras, e uma vez por mês vem um profissional para nos atualizar e tirar dúvidas" (ASB5).

A EPS aproxima a educação da vida cotidiana por meio dos esforços educativos, transformando as atividades laborativas em aprendizagem, por refletir acerca dos problemas vivenciados na prática, reconhecendo o processo de trabalho, sem levar em consideração alguma categoria profissional. 
Dessa forma, articulando o ensino e a prática, direcionando o olhar para diversos contextos educativos e promovendo a formação de sujeitos autônomos. A EPS permite romper tendências que reduzem as práticas educativas em diversas técnicas. Por ela é possível transformar as instituições, por estar inserida no cotidiano de trabalho, proporcionando mudar comportamentos, conceitos e práticas (Fernandes, Cortez, Laprovita, Almeida, Ferreira, \& Corvino, 2017).

Nessa perspectiva, a EPS contribui para transformar as práticas dos profissionais e das instituições de saúde, fazendo do local de trabalho um espaço crítico, reflexivo, comprometido e capacitado. Nesse sentido, contribuindo para relação entre saúde e educação, fortalecendo o processo de formação, de participação, de gestão e de atenção. Fazendo com que cada indivíduo seja agente ativo no processo de reinventar as práticas do cotidiano de trabalho (Ricardi, \& Sousa, 2015).

O estudo de Pinheiro, Azambuja e Bonamigo (2018) evidenciou que os processos educativos facilitam o desenvolvimento da EPS, essencialmente pela utilização de metodologias ativas no cotidiano do trabalho. E outro fator demonstrado foi pela criação do Núcleo de Educação em Saúde pela gestão, responsável por coordenar, articular e organizar os processos pedagógicos da Secretaria Municipal de Saúde (Pinheiro, Azambuja, \& Bonamigo, 2018).

Vale destacar que a EPS desenvolve ações que facilitam o processo de trabalho, sendo fundamental para transformar os modelos de atenção em saúde. Tornando-se uma ferramenta importante para qualificar o trabalho e a gestão em saúde, construindo um SUS voltado para a necessidade de transformar o processo de cuidar e governar em saúde (Pinheiro, Hypólito, \& Kantorski, 2019). Nessa perspectiva, o estudo de D’Ávila et al. (2014) aponta que a adequação do espaço físico facilita o desenvolvimento das atividades de EPS (D'avila, Assis, Melo, \& Brant, 2014).

O processo educativo evidenciado pela EPS é realizado por meio de uma ação política, a partir das práticas vivenciadas pelos trabalhadores envolvidos, que desenvolvem diversas transformações e construções da realidade e do cuidado (Pinheiro, Azambuja, \& Bonamigo, 2018).

O estudo de Ricardi e Sousa (2015) evidenciou a formação de tutores, a adesão por parte dos profissionais, a disponibilidade de recurso, as parcerias, o setor de EPS, e o incentivo às ações como fatores que facilitam o processo de EPS (Ricardi, \& Sousa, 2015).

Contudo, para desenvolver a EPS é necessário considerar os processos educativos que fazem parte das experiências contidas no cotidiano do trabalho, que trazem sentido aos profissionais, aqueles processos que considera os conhecimentos e os fazeres realizados por meio da ação produtiva (Lírio, 2016).

Sob a ótica da Hermenêutica-Dialética e relacionando as definições acima com os fatores que facilitam as atividades de EPS é evidente a necessidade de criar estratégias que favoreçam o processo educativo da EPS nas práticas do cotidiano de trabalho dos profissionais de saúde.

\subsection{Fatores que Dificultam as Atividades de Educação Permanente em Saúde}

Pelos relatos da equipe multiprofissional foi possível identificar os fatores que dificultam as atividades de EPS - incompreensão do processo da EPS, falta de interesse de alguns colaboradores, desconsideração das necessidades dos profissionais pelos gestores e falta de participação da equipe multiprofissional na EPS. 
"Muita gente não tem noção do que seria Educação Permanente em Saúde" (ACS1).

"O que dificulta essa Educação Permanente em Saúde na Estratégia Saúde da Família é a falta de adesão [...], mas eu acredito que o que dificulta muito é o interesse também de alguns colaboradores em participar dessa Educação" (AE1).

"É quando a gestão impõe alguns processos de trabalho e verticaliza algumas [ações coletivas] para os profissionais da saúde não levando em conta as necessidades do território da unidade" (ENF2).

"O não envolvimento de todas as pessoas da equipe da Saúde Família" (MED6).

A EPS é uma ferramenta político-pedagógica que tem como objetivo as dificuldades e os problemas vivenciados no cotidiano de trabalho, proporcionando conhecimento por meio das vivências que envolve profissionais de saúde, gestores, usuários e a população (Carvalho, Alcoforado, \& Santos, 2020).

Nesse sentido, os gestores precisam compreender a EPS como parte integrante do processo de gestão do trabalho em saúde e não apenas como um complemento. A EPS prioriza as práticas em saúde e acontece a partir dos problemas do cotidiano do trabalho, que buscam transformá-las em sua relação e organização. A EPS pode fornecer mudanças na gestão em saúde, por meio das reflexões e críticas construídas por técnicas comprometidas. Portanto, as mudanças que acontecem no processo de trabalho e na APS condizem com as propostas da EPS (Silva, Soder, Petry, \& Oliveira, 2017).

Um estudo evidenciou que a falta de planejamento com os profissionais de saúde são dificuldades encontradas para desenvolverem as atividades de EPS. É necessário que haja continuidade do processo por meio dos indivíduos responsáveis e da gestão, demonstrando a importância e as considerações alcançadas por meio da EPS e possibilitando planejar, envolver e transformar (Weykamp, Cecagno, Vieira, \& Siqueira, 2016).

Vale destacar que as transformações que acontecem no campo da saúde precisam interagir com os diversos setores, como a participação dos atores sociais e da população, a qualificação das organizações em saúde e o investimento na formação e no aprimoramento dos profissionais de saúde. Dessa forma, os envolvidos necessitam construir e compreender o processo que estão vivenciando e experimentando, para que ocorram transformações no cotidiano do trabalho em saúde (Pinheiro, Azambuja, \& Bonamigo, 2018).

Para Ricardi e Sousa (2015), a falta de tempo, a de profissionais na gestão, de investimentos, de compreensão por parte dos profissionais e de estratégias, são dificuldades encontradas para os profissionais desenvolverem as atividades de EPS (Ricardi, \& Sousa, 2015).

Nessa perspectiva, para os profissionais de saúde desenvolverem as atividades de EPS na saúde pública, é necessário que compreendam a realidade do cotidiano do trabalho, conhecendo as dificuldades, as barreiras e as possibilidades, podendo trabalhar de forma acolhedora e eticamente diante dos problemas que possam surgir (Signor, Silva, Gomes, Ribeiro, Kessler, Weiller, \& Peserico, 2015).

Assim, na perspectiva da Hermenêutica-Dialética percebe-se por meio das falas dos participantes da pesquisa os fatores que dificultam as atividades de EPS. Dessa forma, as atividades de EPS precisam desenvolver práticas educativas que evidenciam a problematização do cenário do trabalho, em que os profissionais de saúde são protagonistas desse processo educativo que constrói e descontrói os saberes. 


\section{Conclusões}

A abordagem qualitativa da pesquisa e a fundamentação teórica sustentada pela Hermenêutica-Dialética possibilitou à apresentação dos fatores que apoiam e impedem as atividades de EPS desenvolvidas pela equipe multiprofissional nas USF e que podem subsidiar as adequações necessárias.

As principais facilidades encontradas na pesquisa se referem a potencialidade do trabalho em equipe multiprofissional, no qual a comunicação deve ser de mão dupla, incluindo a gestão e as capacitações devem ocorrer durante o horário de trabalho possibilitando a participação de todos.

Dentre os fatores que dificultam as atividades de EPS estão o desinteresse e a falta de participação da equipe multiprofissional e desconsideração das prioridades da equipe, pela gestão. Essas condições podem gerar a incompreensão conceitual e organizacional da EPS apresentada pelos participantes.

Recomenda-se a estruturação de estratégias que possam viabilizar o desenvolvimento da EPS nas USF do município fundamentados na Lei 8080/90, na PNEPS de 2004, e suas diretrizes de implementação da PNEPS de 2007 e da PNAB de 2017.

\section{Referências}

Ausubel, D. P. A. (1982). A aprendizagem significativa: a teoria de David Ausubel.

Bettoni, R. (2001). Dialética e Sartre: uma possibilidade de se pensar na realidade. Revista Eletrônica Print by FUNREI, 3, 61-70. https://www.ufsj.edu.br/portal2repositorio/File/revistametanoia/numero3/rogeriob.pdf

Carvalho, M. L., Alcoforado, L., \& Santos, E. R. S. (2020). Percepção dos gestores regionais de saúde sobre a política nacional de educação permanente em saúde no Estado do MaranhãoBrasil. Revista Educação e Emancipação, 13(2). http://dx.doi.org/10.18764/23584319.v13n2p228-249

Cardoso, M. F., Santos, A. C. B., \& Alouffa, J. M. L. (2015). Sujeito, linguagem, ideologia, mundo: Técnica hermenêutica-dialética para análise de dados qualitativos de estudos críticos em administração. Revista de Administração Faces Journal, 14(2), 74-93. https://doi.org/10.21714/1984-6975FACES2015V14N2ART2112

D’avila, L. S., Assis, L. N., Melo, M. B., \& Brant, L. C. (2014). Adesão ao Programa de Educação Permanente para médicos de família de um Estado da Região Sudeste do Brasil. Revista Ciência e Saúde Coletiva, 19(2), 401-16. https://doi.org/10.1590/141381232014192.01162013

Fernandes, F. C., Cortez, E. A., Laprovita, D., Almeida, L. P., Ferreira, A. F., \& Corvino, M. P. F. (2017). Educação permanente em saúde sob a perspectiva de Agostinho de Hipona. Revista Brasileira de Enfermagem, 70(3), 656-61. https://doi.org/10.1590/0034-7167-2016-0484

Gerhardt, T. E., \& Silveira, D. T. (2009). Métodos e pesquisa. (1 $\left.1^{a} e d\right)$. UFRGS.

Gilhus, I. S. (2016) Hermenêutica. Revista de estudos da religião, 16(2), 144-56. http://doi.org/10.21724/rever.v16i2.29431

Hermann, N. (2002). Hermenêutica e educação. (1 $\left.{ }^{a} e d\right)$. DP\&A.

IBGE. (2020). Pesquisa de população estimada do munícipio de Assis - SP. https://www.ibge.gov.br/cidades-e-estados/sp/assis.html

Lemos, C. L. P. (2016). Educação Permanente em Saúde no Brasil: educação ou gerenciamento permanente? Revista Ciência e Saúde Coletiva, 21(3), 913-22. http://dx.doi.org/10.1590/1413-81232015213.08182015

Lírio, A. P. S. (2016). O lugar do sensível na educação permanente em saúde: ausência, continuidade ou ruptura. Cadernos de Educação, Tecnologia e Sociedade, Inhumas, 9(3), 375-38. http://dx.doi.org/10.14571/cets.v9.n3.375-383 
Minayo, M. C. S. (2014). O desafio do conhecimento: pesquisa qualitativa em saúde. (14ªed). Hucitec.

Minayo, M. C. S., \& Deslandes, S. F. (2013). Caminhos do Pensamento: epistemologia e método. (3를. Fiocruz.

E-Gestor, Informação e Gestão da Atenção Básica. (2020). Cobertura da Atenção Básica, 2020. https://egestorab.saude.gov.br/paginas/acessoPublico/relatorios/relHistoricoCoberturaAB.xht $\mathrm{ml}$

Organizacion Panamericana de La Salud. (1989). Educación permanente de personal de salud en la Región de las Américas. Fascículo I: Propuesta de reorientación, fundamentos. (2ªed). Washington (D.C): Organización Panamericana de la Salud. (Serie Desarrollo de Recursos Humanos, 78). https://www.paho.org/Spanish/DRH/1740.pdf

Pinheiro, G. E. W., Azambuja, M. S., \& Bonamigo, A. W. (2018). Facilidades e dificuldades vivenciadas na Educação Permanente em Saúde, na Estratégia Saúde da Família. Revista Saúde Debate, 42(4), 187-97. https://doi.org/10.1590/0103-11042018s415

Pinheiro, M. C. C., Hypólito, A. L. M., \& Kantorski, L. P. (2019). Educação permanente no processo de trabalho em saúde mental. Journal of Nursing Health, 9(2). https://docs.bvsalud.org/biblioref/2019/12/1047262/2.pdf

Portaria n. 2.436, de 21 de setembro de 2017. (2017). Aprova a Política Nacional de Atenção Básica, estabelecendo a revisão de diretrizes para a organização da Atenção Básica, no âmbito do Sistema Único de Saúde (SUS). Diário Oficial da União, Brasília, DF. https://bvsms.saude.gov.br/bvs/saudelegis/gm/2017/prt2436_22_09_2017.html

Ricardi, L. M., \& Sousa, M. F. (2015). Educação permanente em alimentação e nutrição na Estratégia Saúde da Família: encontros e desencontros em municípios brasileiros de grande porte. Revista Ciência e Saúde Coletiva, 20(1), 209-18. https://doi.org/10.1590/141381232014201.20812013

Severino, J.A. (2016). Metodologia do trabalho científico. (24ㄹa ed). Cortez.

Soratto, J., Pires, D. E. P., Dornelles, S., \& Lorenzetti, J. (2015). Estratégia saúde da família: uma inovação tecnológica em saúde. Texto \& Contexto Enfermagem, 24(2), 584-92. https://doi.org/10.1590/0104-07072015001572014

Signor, E., Silva, L. A. A., Gomes, I. E. M., Ribeiro, R. V., Kessler, M., Weiller, T. H., \& Peserico, A. (2015). Educação Permanente em Saúde: desafios para gestão em Saúde Pública. Revista de Enfermagem UFSM, 5(1), 01-11. https://doi.org/10.5902/2179769214766

Silva, L. A. A., Soder, R. M., Petry, L., \& Oliveira, I. C. (2017). Educação permanente em saúde na atenção básica: percepção dos gestores municipais de saúde. Revista Gaúcha de Enfermagem, 38(1). https://doi.org/10.1590/1983-1447.2017.01.58779

Weykamp, J. M., Cecagno, D., Vieira, F. P., \& Siqueira, H. C. H. (2016). Educação Permanente em Saúde na Atenção Básica: percepção dos profissionais de Enfermagem. Revista de Enfermagem da UFSM, 6(2), 281-89. https://doi.org/10.5902/2179769216754 